Bulletin of Mathematical Biology (2000) 62, 1087-1108

doi:10.1006/bulm.2000.0199

Available online at http://www.idealibrary.com on IDE

\title{
A Unifying Theory of Refractive Error Development
}

\author{
GEORGE K. HUNG* \\ Department of Biomedical Engineering, \\ Rutgers University, \\ 617 Bowser Road, \\ Piscataway, \\ NJ 08854-8014, U.S.A. \\ E-mail: shoane@rci.rutgers.edu
}

KENNETH J. CIUFFREDA

Department of Vision Sciences,

State College of Optometry, State University of New York,

33 West $42^{\text {nd }}$ Street,

New York, NY 10036,

U.S.A.

E-mail: kciuffreda@sunyopt.edu

\begin{abstract}
While retinal defocus is believed to be myopigenic in nature, the underlying mechanism has remained elusive. We recently constructed a theory of refractive error development to investigate its fundamental properties. Our Incremental RetinalDefocus Theory is based on the principle that the change in retinal-defocus magnitude during an increment of genetically-programmed ocular growth provides the requisite sign for the appropriate alteration in subsequent environmentally-induced ocular growth. This theory was tested under five experimental conditions: lenses, diffusers, occlusion, crystalline lens removal, and prolonged nearwork. Predictions of the theory were consistent with previous animal and human experimental findings. In addition, simulations using a MATLAB/SIMULINK model supported our theory by demonstrating quantitatively the appropriate directional changes in ocular growth rate. Thus, our Incremental Retinal-Defocus Theory provides a simple and logical unifying concept underlying the mechanism for the development of refractive error.
\end{abstract}

(C) 2000 Society for Mathematical Biology

\section{INTRODUCTION}

During infancy, there is an inherent mismatch between the optical power of the cornea/lens and the axial length of the eyeball (Scammon and Armstrong, 1925). Yet, as the normal eye matures, the cornea/lens and ocular tunics begin to develop in a concerted manner to provide a relatively precise focused image on the retina (Bennett and Rabbetts, 1989). This process is called emmetropization

\footnotetext{
*Author to whom correspondence should be addressed.

$0092-8240 / 00 / 061087+22 \quad \$ 35.00 / 0$

(C) 2000 Society for Mathematical Biology
} 
(Yackle and Fitzgerald, 1999). Clearly, certain critical information is used to link cornea/lens and axial growth. One of the most important cues for regulating axial growth is retinal-image blur (McBrien and Millodot, 1986; Ong and Ciuffreda, 1997; Wallman, 1997; Norton, 1999), which is dependent on the interaction of both cornea/lens and axial length. Cornea/lens growth and its consequent change in optical power will alter retinal-image blur, but an appropriate change in the axial length growth rate will act to reduce this blur, and in turn restore the balance between these two components. It would seem that simple visual feedback related to retinal-image blur could provide the requisite control signal to regulate axial growth. However, such appropriate changes in growth rate occur even when the optic nerve is severed (Troilo et al., 1987; Wildsoet and Pettigrew, 1988) or the midbrain nuclei for controlling accommodation are lesioned (Troilo, 1989), thus precluding any visual feedback mechanism. Moreover, since blur per se is an even-error signal (Stark, 1968), it lacks the requisite directional sensitivity for controlling axial growth. Indeed, for this reason, the mechanism underlying myopic development has remained a puzzle for decades.

The presence of refractive error can be considered to represent a failure of the emmetropization process (Yackle and Fitzgerald, 1999). Both genetic and environmental factors may be involved (McBrien and Millodot, 1986; Gwiazda et al., 1993; Jiang and Woessner, 1996; Ong and Ciuffreda, 1997). An important environmental factor is chronic exposure to retinal defocus related to nearwork. The inability to regulate and reduce blur over an extended period of time (i.e., months or years) could result in the development of myopia. And, since myopia affects $25 \%$ of the adult population in the United States (Sperduto et al., 1983) and 75\% or more of adults in Asian countries such as Taiwan (Lin et al., 1996), it poses a major world-wide public health concern.

Numerous studies have attempted to determine the effect of various visual manipulations on induced ocular growth and refractive development. The findings have been mixed with respect to the resultant direction of refractive shift. Some manipulations produced a myopic shift. These included: prolonged nearwork (Grosvenor and Goss, 1999), graded diffusers (Smith and Hung, 2000), and black-occluder contact lenses (Tigges et al., 1990; Iuvone et al., 1991). On the other hand, other manipulations resulted in a hyperopic shift. These included: very strong diffusers (O’Leary et al., 1992; Bradley et al., 1996), crystalline lens removal (Wilson et al., 1987), and initial imposition of graded diffusers (Smith and Hung, 2000). Finally, manipulations using plus or minus lenses in the chick (Schaeffel et al., 1990), tree shrew (Norton, 1999; Siegwart and Norton, 1999), and monkey (Smith and Hung, 1999) resulted in either hyperopic or myopic growth, respectively.

It has been proposed that dopamine, a neuromodulator released by amacrine and interplexiform cells, is involved in the regulation of ocular growth (Hung and Ciuffreda, 2000b). For example, the amount of axial elongation has been found to be dependent on the dopamine level (Stone et al., 1989; Yackle and Fitzgerald, 1999). Also, Bjelke et al. (1996) found that amacrine and/or interplexiform cells, 
with their sparse branches in the outer plexiform layer, operate via volume transmission to influence the outer plexiform layer and outer segment, as well as other layers of the retina. Such a diffusion process in the extracellular space allows neuromodulators in the extra-retinal matrix, via its effect on proteoglycan synthesis, to modulate the rate of scleral growth. Indeed, results from animal experiments have shown that elevated levels of proteoglycan synthesis were associated with increased sclera thickness and dry weight (i.e., decreased rate of axial elongation) (Gottlieb et al., 1990; Christiansen and Wallman, 1991; McBrien et al., 1999).

Various descriptive as well as quantitative models have been proposed to account for different aspects of refractive error development and emmetropization (Medina, 1987; Schaeffel and Howland, 1988; Bartmann and Schaeffel, 1994; Flitcroft, 1998; Blackie and Howland, 1999; Hung and Ciuffreda, 1999; Norton, 1999). However, these models have not been able to explain the fundamental underlying mechanism of refractive error development.

To explore the underlying mechanism and to account for the apparently mixed experimental findings, a recent theory of refractive error development constructed by us (Hung and Ciuffreda, 2000a,b) was used and expanded. Two fundamental insights underlie our theory, called the Incremental Retinal-Defocus Theory. First, local retinal-defocus magnitude is critical in the development of environmentally induced refractive error (Goss and Wickham, 1995). Second, manipulations of the optical environment are effective in producing refractive error mainly during the ocular growth and maturational period [in contrast, most mature adult animals are relatively insusceptible to optical manipulations (Goss and Winkler, 1983; Adams and McBrien, 1992; Siegwart and Norton, 1999)]. Each insight alone is insufficient to provide a workable bidirectionally-sensitive theory, but when these two are combined, they provide a coherent framework for a unifying theory of refractive error development. Our theory is based on the concept that the change in magnitude of retinal defocus during an increment of genetically-programmed axial length growth provides the critical information for directional modulation of growth rate (described in Section 3.1). The term genetically-programmed is used to describe the normally occurring ocular growth that has been pre-programmed genetically, which should be distinguished from environmentally-induced growth that is due to change in retinal defocus. Both involve neuromodulator release, with the environmentally-induced component in effect modulating the normal geneticallyprogrammed release rate. In the present paper, this theory was tested under five critical experimental conditions to assess the generality of the proposed underlying mechanism. In addition, a MATLAB/SIMULINK model was constructed to demonstrate quantitatively the direct effect of change in retinal defocus, via signal cascade through the retinal layers, on scleral growth rate. 


\section{Methods}

The development of a theory for the control of ocular growth rate requires the conceptual linking of a number of areas including: cornea and lens growth, optics of the eye, retinal neural signal processing, scleral growth, and ocular neurochemistry (Ong and Ciuffreda, 1997). The fundamental principles can be understood in terms of answers to three critical questions: (i) What is the contribution of the cornea and lens to the emmetropization process? (ii) How do retinal neurochemicals process the retinal-defocus information?; and (iii) How is this information used to regulate the rate of ocular growth?

The present theory, called the Incremental Retinal-Defocus Theory, was conceptualized from the results of our recent comprehensive quantitative model (Hung and Ciuffreda, 1999) and developed in the form of a schematic representation (Hung and Ciuffreda, 2000a,b). The schematic model was analysed under the conditions of: the imposition of plus or minus lenses, graded strength of diffusers, black occluder, removal of the crystalline lens, and prolonged nearwork.

In addition to the schematic model, a quantitative and homeomorphic model of the local retinal circuitry was constructed using MATLAB (5.3)/SIMULINK (3.0), wherein the various components and interconnections have a direct correspondence with known retinal anatomy and physiology (Dowling, 1996) [see Fig. 6(b)]. The retinal layers (outer to inner) are arranged from left to right, and they consist of photoreceptors, outer plexiform layer, bipolar cell layer, inner plexiform layer, and ganglion cell layer. Two pathways, the transient [center photoreceptor, center bipolar $\mathrm{A}$, and transient ganglion (represented by a $50 \mathrm{~Hz}$ high-pass filter)], and the sustained (center photoreceptor, center bipolar B, and sustained ganglion). Each pathway consists of a center-surround organizational structure, with the horizontal cells relaying surround information to center bipolar B and then to the sustained ganglion cells, and the amacrine cells (represented by a $50 \mathrm{~Hz}$ high-pass filter) relaying change in surround information to center bipolar $\mathrm{A}$, and in turn to the transient ganglion cells. The output of center bipolar B, which represents retinaldefocus amplitude, is first rectified to retain only its magnitude. This signal passes through two low-pass filters that represent the conversion from neural signal to neuromodulator release. These low-pass and high-pass filters are assumed to be part of the neural circuitry in the inner plexiform layer, most likely involving amacrine cells. Neuromodulator release rate is modeled as a low-pass filter with a time constant of $1 \mathrm{~h}$ (which is consistent with Smith and Hung, 2000), and this represents the diffusion process of release from the inner plexiform layer in the retina to the choroid. The diffusion through the choroid is represented by a low-pass filter with a time constant of $10 \mathrm{~h}$. The proteoglycan synthesis rate is model as a low-pass filter with a time constant of $20 \mathrm{~h}$. Finally, the inverter converts a decrease in proteoglycan synthesis rate to an increase in scleral growth rate. An important part of the model is the interplexiform feedback regulation of horizontal cell gain. The horizontal gain control is regulated using a tanh function, which compresses and 
limits the interplexiform input signal to be between -0.5 and +0.5 . The resultant signal is subtracted from the nominal horizontal gain $(0.5$ for each simulated horizontal cell) to provide the combined horizontal cell gain. Thus, for example, larger retinal-defocus signal from the interplexiform neurons results in decreased horizontal cell gain. Such regulation results in a relatively constant sensitivity to changes in retinal-defocus magnitude, and thereby precludes the need for a memory mechanism.

The stimulus amplitude was defined in terms of its relative brightness over a unit of retinal area, in which a unit area represents the extent of the limit of visual acuity [about $1 \mathrm{~min}$ of arc linear dimension (Westheimer, 1981)]. For simplicity, each center or surround unit of retinal area could be assigned only one of three brightness levels: $-1,0$, or 1 . The contribution from an additional unit of spatially extended surround area could be included by adding its brightness contribution to that from the immediately adjacent surround area. In this way, the surround amplitude reflected the relative amount of retinal defocus rather than retinal-image contrast per se. To simulate the temporal variation in brightness over a particular retinal locus in the course of a normal viewing period, all retinal areas received a $0.1 \mathrm{~Hz}$ square-wave signal. Thus, for example, the receptive field center would always consist of a \pm 1 amplitude peak-to-peak (ptp) signal (i.e., a $0.1 \mathrm{~Hz}$ squarewave with amplitudes ranging from -1 to +1 ). On the other hand, the surround signal could vary depending on the amount of modulation $(0,1$, or 2$)$, which represents the amount of retinal defocus. For example, a modulation of 0 (i.e., zero amplitude for the surround) represents a relatively small amount of retinal defocus (it is not zero retinal defocus because of the limit to the resolution of the retinal area), or conversely, a relatively sharply focused image. A modulation of 1 from one unit area of surround (i.e., a $0.1 \mathrm{~Hz}$ square-wave with amplitude ranging from -1 to +1 ) represents a moderate amount of retinal defocus. Finally, a modulation of 2, due to the summing of two adjacent unit surround areas (i.e., a $0.1 \mathrm{~Hz}$ squarewave with amplitudes ranging from -2 to +2 ) represents a relatively large amount of retinal defocus.

The quantitative model was tested using a selected series of surround modulation steps $(0,1,2,1,0)$ at $100 \mathrm{~h}$ intervals over a $500 \mathrm{~h}$ period. This was chosen to demonstrate transitions between various levels of retinal-defocus magnitude. More importantly, the model simulation was used to demonstrate the long-term effects of changes in retinal-defocus magnitude on scleral growth rate. Various model parameters were monitored: the horizonal cell gain (representing feedback modulation from inner to outer plexiform layer by interplexiform neurons), the rates of neuromodulator release and proteoglycan synthesis, and the relative change in axial length. These data were stored in a Gateway-2000 computer and plotted on a Laserjet 4 printer. 


\section{Results AND Discussion}

3.1. Basic principles of the theory. The answers to the posed questions for the theory of refractive error development have been discussed in detail elsewhere (Hung and Ciuffreda, 2000a,b) and are overviewed briefly below.

(1) Corneal growth does not contribute to the emmetropization process after age 2 years. During the first two years of life, the cornea and axial length overcome the initial hyperopia and grow rapidly and in concert as part of the emmetropization process (Scammon and Armstrong, 1925; York and Mandell, 1969; Weale, 1982). Thereafter, corneal power remains relatively stable (Sorsby et al., 1961; Garner et al., 1990; Goss and Jackson, 1993; Fledelius and Stubgaard, 1986), until the adult years, when it may increase slightly (Fledelius and Stubgaard, 1986; Grosvenor and Goss, 1998) Since corneal flattening rather than steepening would be needed to compensate optically for the axial length growth, it is clear that the cornea plays little or no role in the emmetropization process after the first 2 years of life (Sorsby et al., 1961; Goss and Erickson, 1987; Goss and Jackson, 1993; Fledelius and Stubgaard, 1986). Moreover, since there is no evidence that visual feedback plays a role in the growth of the lens (Sorsby et al., 1961; Van Alphen, 1961), emmetropization during this later period involving any large artificially induced retinal-image defocus must be provided only by the rate of change of axial length growth.

(2) Neuromodulators control sensitivity to changes in retinal-image contrast. In contrast to neurotransmitters, such as glutamate, acetylcholine, and GABA, which respond rapidly to retinal stimulation (Dowling, 1996), neuromodulators, such as dopamine, seratonin, and neuropeptides (Dowling, 1996), act over a longer period, and in addition, may cause changes in the neuronal synapses (Windhorst, 1996). An example of synaptic plasticity in the retina can be seen in the interplexiform cells in the retina (Dowling, 1996). These neurons, which contain dopamine, receive their inputs from the amacrine cells in the inner plexiform layer, and then send their outputs back to the horizontal cells in the outer plexiform layer (Cohen, 1981; Dowling, 1996). Dopamine serves as a neuromodulator by altering the properties of the horizontal cell membrane and decreasing the flow of current across the membrane (Dowling, 1996; Windhorst, 1996). Moreover, because of the center-surround structure of the retina, the interplexiform neurons respond in a graded manner to local retinal-image contrast (Cohen, 1981; Dowling, 1996).

We have proposed that feedback regulation provided by the interplexiform neurons from the inner to outer plexiform layers maintains a relatively constant sensitivity to retinal-image contrast, and that such interplexiform neuronal activity leads to a corresponding change in the neuromodulators (Hung and Ciuffreda, 2000a,b). Such feedback regulation is useful because it would preclude the need for a memory mechanism to register and store previous levels of retinal defocus for the pur- 
poses of update and comparison. The release of neuromodulators results in synaptic changes in the horizontal cells (Dowling, 1996; Windhorst, 1996). This in turn alters retinal sensitivity to center-surround input, which helps to shift the steadystate operating level to permit responsivity to transient changes in local retinalimage contrast. Thus, the net rate of release of neuromodulators is not dependent on the absolute level of retinal defocus, but rather on the change in retinal-defocus magnitude. The release of neuromodulators also causes structural changes in the sclera via modulation of proteogly can synthesis (Rada et al., 1992; Norton and Rada, 1995), wherein an increase in proteoglycan synthesis rate results in a greater structural integrity of the sclera, and in turn, a decrease in axial growth rate relative to normal; and conversely, a decrease in proteogly can synthesis rate results in less structural integrity of the sclera, and in turn, an increase in axial growth rate relative to normal (Christiansen and Wallman, 1991; Marzani and Wallman, 1997; Wildsoet, 1998; McBrien et al., 1999; Siegwart and Norton, 1999).

(3) The overall mechanism for regulating the rate of axial length growth.

Genetically-programmed mechanisms determine a baseline rate of neuromodulator release that is associated with the normal axial growth rate. Retinal defocusinduced changes in the rate of neuromodulator release are superimposed onto this baseline level to result in changes relative to the normal axial growth rate. The net effect of the local-retinal mechanism, as discussed above, is that the change in retinal-defocus magnitude, and in turn the change in the rate of neuromodulator release, are in opposite directions with respect to the change in the rate of defocus-induced axial growth relative to normal. Thus, during an increment of genetically-programmed ocular growth, the change in retinal-defocus magnitude due to the incremental change in ocular geometry provides the directional information needed to modulate the rates of release of neuromodulators and proteoglycan synthesis, which in turn produce structural changes in the sclera for regulation of ocular growth (Wildsoet, 1998; Siegwart and Norton, 1999). For example, during an increment of genetically-programmed ocular growth (over days), if the retinaldefocus magnitude decreases, the axial growth rate increases. This results in relative myopic growth. On the other hand, if the retinal-defocus magnitude increases, the axial growth rate decreases. This results in relative hyperopic growth. These resultant axial growth rate changes are consistent with the emmetropization process.

\subsection{Applications of the theory.}

Lenses. During ocular development, the eye exhibits continuous geneticallyprogrammed growth. The imposition of a lens causes changes in retinal defocus which act to modulate the genetically-predetermined normal growth rate, and thereby alters the overall axial growth rate. This modulation can be illustrated by the following example. Consider the effect of introducing spherical lenses in front of the eye. The change in size of the blur circle during a small increment 
(a)

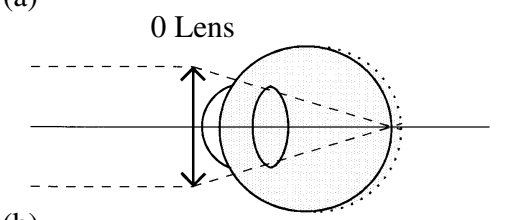

(b)

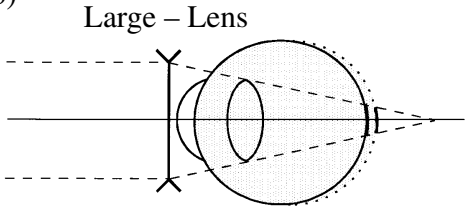

(c)

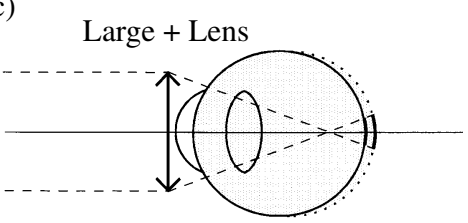

Figure 1. Schematic representation of change in blur circle during a small increment of normal genetically-programmed ocular growth under the conditions of: (a) zero lens; (b) minus lens; and (c) plus lens.

of normal genetically-programmed ocular growth for large imposed zero, minus, and plus lenses is shown schematically in Fig. 1(a)-(c), respectively. A neuromodulator, such as dopamine, maintains a certain level of neuronal activity related to retinal-image contrast by means of the local retinal feedback mechanism described earlier. The net effect is that the rate of neuromodulator release is dependent not on the absolute level of retinal-defocus magnitude, but rather on the change in retinal-defocus magnitude during the increment of genetically-programmed ocular growth, as was also mentioned earlier. For example, for a zero power lens, there is no change in the size of the blur circle. Thus, no additional neuromodulator is released, and the normal genetically-based incremental axial growth pattern of the young eye is maintained. With the introduction of a minus lens, however, the size of the blur circle is decreased; thus, the rates of neuromodulator release and in turn proteoglycan synthesis are decreased, thereby resulting in an increase in axial growth rate. On the other hand, with the introduction of a plus lens, the size of the blur circle is increased; thus, the rates of neuromodulator release and in turn proteoglycan synthesis are increased, thereby resulting in a decrease in axial growth rate. Hence, either a decrease or increase in mean retinal-defocus magnitude during an increment of genetically-programmed axial growth is proposed to cause a change in the rate of neuromodulator release, which in turn leads to structural changes in the sclera (Wildsoet, 1998; Siegwart and Norton, 1999), that are manifest as appropriate changes in the rate of axial growth and reflect the active emmetropization process. 
(a)

(b)
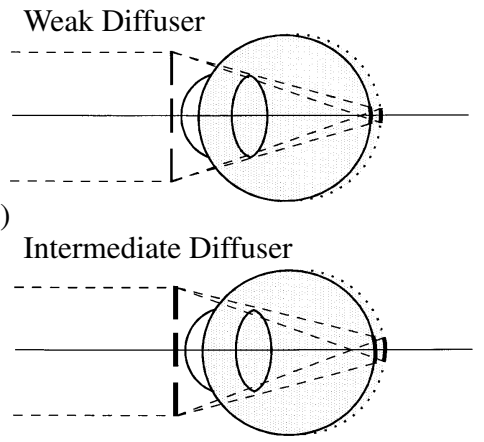

(c)

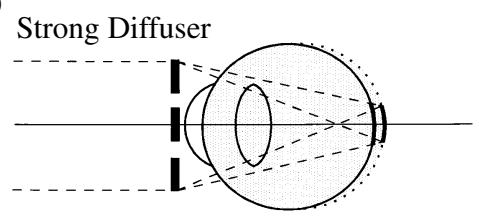

Figure 2. Schematic representation of the change in blur circle during small increment of normal growth under the conditions of: (a) weak diffuser; (b) intermediate diffuser; and (c) strong diffuser.

Graded diffusers. The theory can also be applied to recent experimental results on the effect of graded diffusers in monkeys (Smith and Hung, 2000). Although a diffuser can have complex optical effects (Smith and Atchison, 1997), its primary effect is to disperse or scatter the rays of light that are transmitted through the diffuser. This is schematically represented by a cone of light and seen in the figure as two lines representing the boundaries of the cone [Fig. 2(a)-(c)]. The angle is increased for a stronger diffuser to represent its greater dispersional effect [Fig. 2(c)]. This results in a blur circle on the retina. Since accommodation would be relatively imprecise under this condition, for simplicity, the focal point for the central ray of the cone of diffused light is set midway between the two incremental foveal positions along the visual axis. It can be shown that based on the geometrical configuration using ray tracing, for the weak diffuser with small dispersion [Fig. 2(a)], the decrease in retinal-defocus magnitude during the genetically-programmed incremental growth is relatively minor. Thus, the decrease in neuromodulator and proteoglycan synthesis is small, thereby resulting in a relatively small increase in axial growth rate. On the other hand, for the stronger diffusers with stronger dispersions [Fig. 2(b) and (c)], there is a progressively greater decrease in retinal-defocus magnitude. Therefore, the intermediate diffuser will result in an intermediate increase in axial growth rate, whereas the strong diffuser will result in the largest increase in axial growth rate. These findings are consistent with experimental results (Smith and Hung, 2000). 
(a)

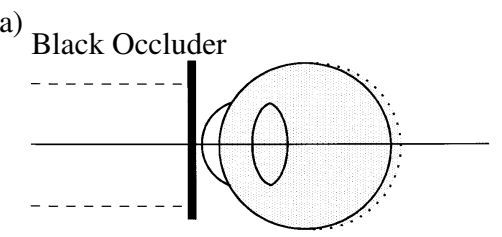

(b)

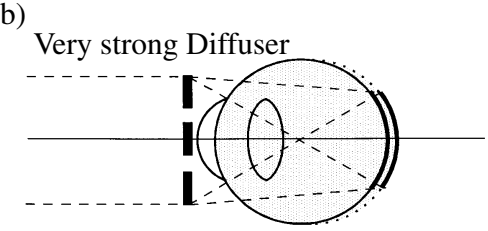

(c) Remove Crystalline Lens

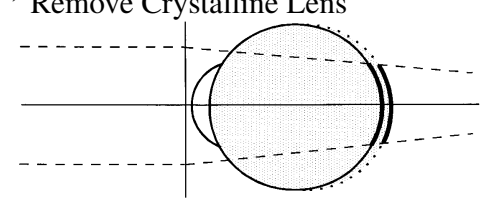

Figure 3. Schematic representation of the change in blur circle during small increment of normal growth under the conditions of: (a) black occluder; (b) very strong diffuser; and (c) removal of crystalline lens.

Black occluder. For the more extreme condition of a full black occluder (Smith and Hung, 2000), there is a complete absence of form vision along with a drastically reduced retinal luminance level. Our theory predicts that as a result of the absence of retinal signal-induced feedback regulation of horizontal cells in the dark (Dowling, 1996), there would likewise be a drastic reduction in the rate of neuromodulators, and in turn a substantial decrease in the rate of proteoglycan synthesis [Fig. 3(a)]. This will result in a marked increase in the axial growth rate and the development of myopia, which is consistent with experimental results (Tigges $e t$ al., 1990; Iuvone et al., 1991).

Very strong diffuser or removal of crystalline lens. For the condition of either a very strong diffuser [Fig. 3(b)] or the removal of the crystalline lens [Fig. 3(c)], there is a large initial increase in blur magnitude. Our theory predicts that this will cause an initial increase in the rates of neuromodulators and proteoglycan synthesis, which will result in a decrease in the axial growth rate, and therefore relative hyperopia. However, the large blur magnitude, with its boundary rays being nearly parallel, does not change substantially during the subsequent time increments. This lack of change in retinal-defocus magnitude will result in a normal (i.e., determined by genetic preprogramming) rate of neuromodulator release, and therefore the subsequent axial growth rate will be nearly normal. Thus, the initial hyperopia is retained (Smith and Hung, 2000). 
(a)

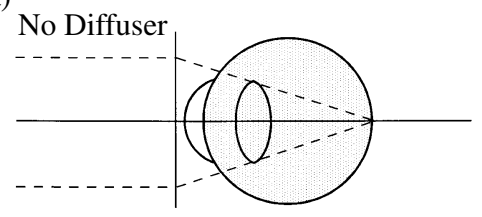

(b)

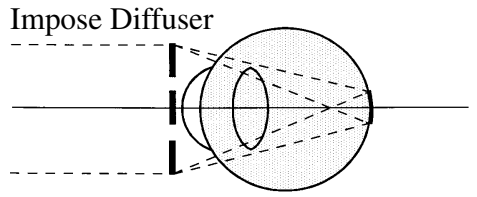

Figure 4. Schematic representation of (a) the initial normal condition; and (b) the transient effect due to the imposition of a diffuser.

Transient hyperopia. Transient hyperopia following the imposition of a diffuser can be analysed as follows (Fig. 4). Prior to the imposition of the diffuser, the retinal-defocus magnitude is near or below the threshold level. Thus, there is a relatively small amount of retinal defocus-induced neuromodulator release and proteoglycan synthesis, and the growth rate is determined primarily by genetic factors. However, the sudden imposition of the diffuser results in an immediate increase in retinal-defocus magnitude. Thus, there is a transient increase in the rates of neuromodulators and proteoglycan synthesis. This results in a decrease in axial growth rate, or a transient relative hyperopia. However, subsequently, as the geneticallyprogrammed growth continues, the condition becomes similar to that shown in Fig. 2(a)-(c). Since the boundaries of the light bundles from the diffuser converge beyond the retina, there will now be an incremental decrease in retinal-defocus magnitude. The resultant decrease in the rates of neuromodulators and proteoglycan synthesis, and in turn increase in axial growth rate, will first effectively null out the initial hyperopia and then eventually develop into myopia [see Fig. 2(a)-(c)]. This accounts for the transient nature of the initial hyperopia seen in some of the recent monkey experimental results (Smith and Hung, 2000).

The initial transient increase in retinal-defocus magnitude may also explain some recent findings of a greater effect of plus over minus lenses in controlling ocular growth. For example, Wildsoet and Collins (2000) found that imposition of a plus/minus multifocal lens in chicks results in a preference towards hyperopic ocular development, which indicates a preference of plus over minus lens. Also, Winauer et al. (2000) found that chicks compensated to imposed plus lenses even when blurred with diffusers, suggesting a particular potency of myopic blur in changing ocular growth rate. These results can be explained by the fact that the imposition of either a plus or a minus lens will cause an initial transient increase in retinaldefocus magnitude. During a subsequent increment of genetically-programmed 
growth, the plus lens will cause an increase in retinal-defocus magnitude [see Fig. 1(c)], which augments the initial transient increase. On the other hand, the minus lens will cause a decrease in retinal-defocus magnitude [see Fig. 1(b)], which opposes the initial transient increase. The net result is a greater effect of plus over minus lens during the initial phases of ocular development following such optical manipulations.

Prolonged nearwork. Finally, the theory can be analysed for the condition of prolonged nearwork, as in the case of the development of school myopia, wherein relatively small amounts of retinal defocus are present over extended periods of time (i.e., weeks or months) (Ong and Ciuffreda, 1997; Rosenfield and Gilmartin, 1998). This can be understood by examining the normal accommodative stimulus/response (AS/R) function (Ciuffreda and Kenyon, 1983; Ciuffreda, 1991, 1998; Ong et al., 1993; Hung, 1998) (Fig. 5). This function is an s-shaped curve showing slight over-accommodation at distance and progressive under-accommodation at near with increased dioptric demand. Thus, during nearwork, which is represented by a relatively large accommodative stimulus (e.g., 4D), the accommodative response lags the stimulus, which results in chronic hyperopic defocus (Fig. 5). Let the near target be at a fixed distance at $25 \mathrm{~cm}$, so that the accommodative stimulus is $4 \mathrm{D}$, and the response is slightly smaller. Following an increment of normal genetically-programmed axial length growth, the effective axial length optical power will have decreased, so that the effective accommodative stimulus (or the optical power needed to focus onto the retina of the now incrementally lengthened eyeball) will have also decreased. Thus, less accommodative response would now be necessary for clear retinal imagery. This is equivalent to moving slightly downward and to the left on the AS/R function. The decrease in accommodative error is now associated with a decrease in defocus blur (Fig. 5). In addition, according to the same arguments above regarding large imposed minus lens [Fig. 1(b)], there would be a slight decrease in the rates of neuromodulator release and proteoglycan synthesis. This in turn would result in an increase in axial growth rate relative to normal, i.e., myopic growth, which is consistent with the emmetropization process. Moreover, if the retinal defocus is left uncorrected over a prolonged period of time, the process would repeat itself and effectively continue to move slightly down and to the left on the AS/R curve, which would eventually lead to continued increase in axial growth rate relative to normal, and in turn a progressive development of myopia (Abbott et al., 1998). This process is similar to that for the imposition of a minus lens [Fig. 1(b)], except in this case it is the shape of the AS/R curve (Ong et al., 1993; Hung, 1998) (i.e., the relationship between the curve and the $1: 1$ line) (Fig. 5) that drives the increase in axial growth rate and subsequent myopia development. It is somewhat ironic that rather than being a failure of the emmetropization process, myopia development is actually a result of the emmetropization process that operates under the constraints of the AS/R function during increments of genetically-programmed ocular growth. 


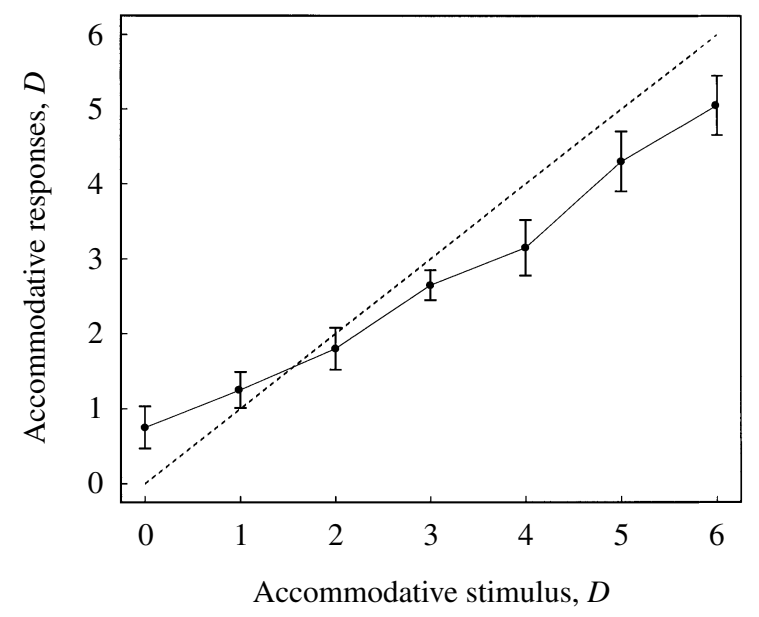

Figure 5. Plot of averaged accommodative stimulus-response data for 10 visually normal subjects. Filled circles and error bars represent group mean \pm SEM. Adapted from Ong et al. (1993) with permission from the authors.

The analysis above also explains why the increase in axial growth rate is primarily associated with prolonged nearwork rather than farwork. The reduction in accommodative error during an increment of genetically-programmed ocular growth is more pronounced for near (4D) than for far $(<1 \mathrm{D})$ viewing (see Fig. 5). Furthermore, some individuals (hyperopes and emmetropes) may not develop myopia subsequent to nearwork because they may have higher thresholds for inducing axial length growth than the myopes (Hung and Ciuffreda, 1999).

In addition, according to the theory, the rate of ocular growth is dependent on the change in retinal-defocus magnitude regardless of how it is generated. Therefore, in the absence of an increment of genetically-programmed ocular growth, retinal-defocus induced axial elongation due to prolonged nearwork can still occur as long as the individual exhibits susceptibility to neurochemical influence on scleral growth. This may explain the finding of form deprived myopia in adolescent animals even after they are past the rapid juvenile growth phase (Troilo et al., 2000b).

3.3. Basic retinal anatomy and physiology. Signals are transmitted in the retina through three types of neurons: photoreceptors, bipolar cells, and ganglion cells. The photoreceptors (rods and cones) are stimulated by light and relay the signal through bipolar cells, which in turn relay the information through the ganglion cells. The axons of the ganglion cells in the retina form the optic nerve, which transmits retinal-image information to the higher cortical centers. Bipolar cells also receive light stimulus information from neighboring, or surround, photoreceptors via lateral connections from horizontal cells in the outer plexiform layer. This center-surround organizational structure provides local retinal-image contrast 
information to the 'sustained' ganglion cells, which respond to sustained contrast information. On the other hand, 'transient' ganglion cells respond to change in the surround via amacrine cells in the inner plexiform layer. Thus, these neurons relay information regarding the change in retinal-defocus magnitude. In addition, interplexiform neurons from the inner to the outer plexiform layer modulate the long-term sensitivity of horizontal cells to surround input. Thus, this feedback mechanism serves to adjust the steady-state sensitivity level to provide relatively constant sensitivity to changes in local contrast (Dowling, 1996).

3.4. Quantitative MATLAB/SIMULINK model. A conceptual block diagram of the model is shown in Fig. 6(a). It is based on the principle that the magnitude of retinal defocus can be represented by the difference in center and surround excitation. A change in this signal, and thus retinal-defocus magnitude, provides the requisite sign for modulating ocular growth. The sensitivity to local retinal-image contrast is maintained at a relatively constant level by means of feedback regulation of horizontal cell gain provided by the interplexiform neurons. This precludes the need for a memory mechanism for storing information regarding the previous level of retinal-defocus magnitude, so that its change can be discerned. The release of neuromodulator in turn results in changes in the rate of scleral proteoglycan synthesis, which causes a change in scleral growth rate. This relative growth rate is added to the ongoing normal genetically-programmed ocular growth rate to provide the overall axial length growth.

The detailed model is shown in Fig. 6(b). The sustained pathway consists of the photoreceptor, bipolar, and sustained ganglion cells. It is modulated by surround signals via horizontal cells in the outer plexiform layer to provide local steady-state or sustained contrast information. The transient pathway also consists of photoreceptor, bipolar, and transient ganglion cells. However, it is modulated by surround signals via amacrine cells in the inner plexiform layer to provide information regarding change or transients in local contrast. Feedback regulation is provided by the interplexiform neurons that receive signals for neuromodulator release in the inner plexiform layer and modulate the gain of horizontal cells in the outer plexiform layer to maintain a relatively constant sensitivity to change in local contrast. The center bipolar cell receives a signal derived from the difference between center and summed surround inputs, which thus represents the summated amount of retinal-image defocus across the overlapping, spatially contiguous center and surround receptive field area. This signal is differentiated by neural circuitry in the inner plexiform layer, which most likely contains amacrine cells. This change is rectified, so that the 'envelope' of the signal, which represents the overall change in retinal-defocus magnitude, drives the rate of neuromodulator release. The neuromodulator, or a cascade of neurochemicals related to the release of the neuromodulator (Wallman, 1997), passes through the choroid to reach the sclera. The transit of the neuromodulator through the choroid may result, at least in the monkey, in a volume change that is observed as a change in choroidal thick- 

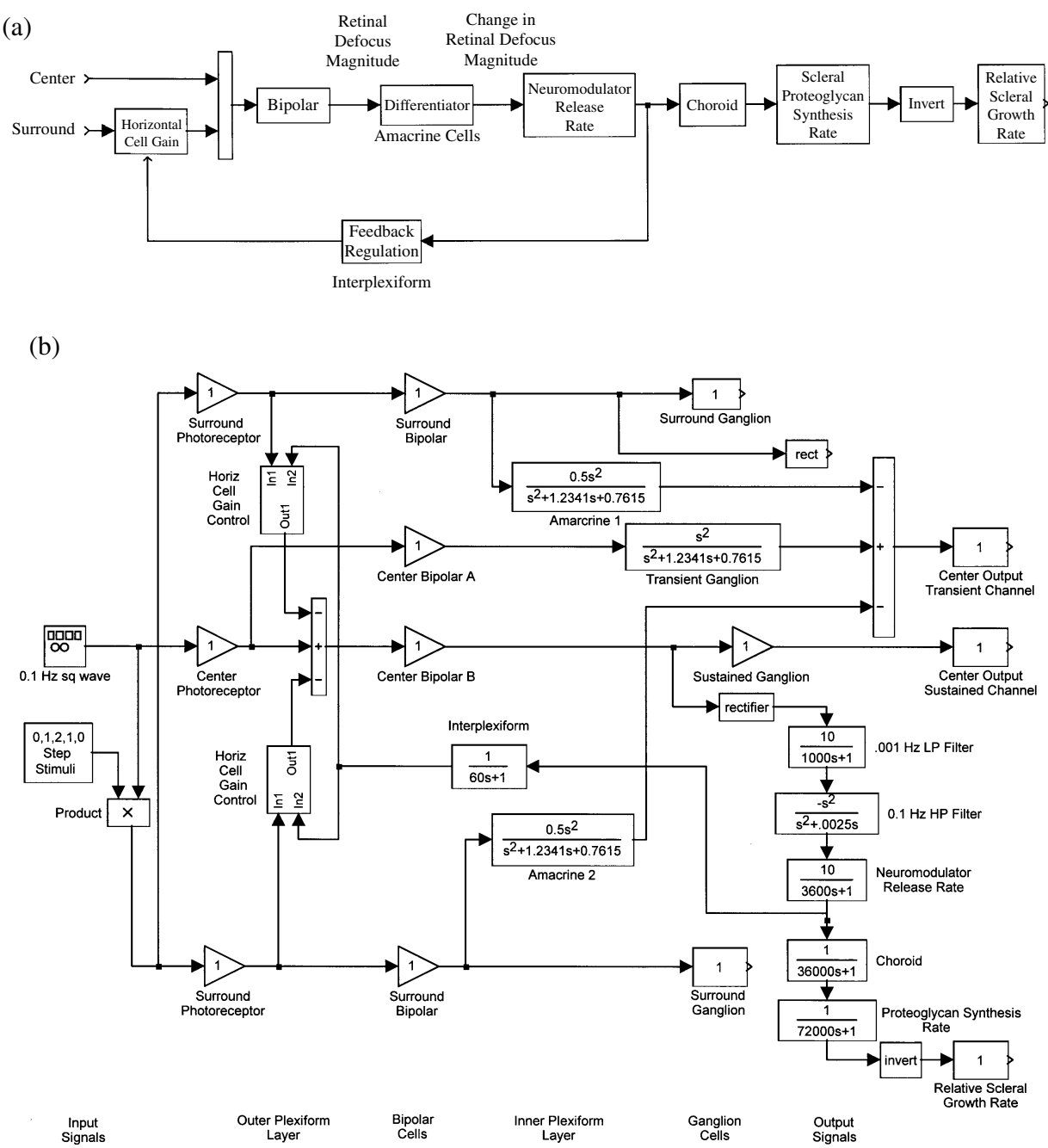

Figure 6. (a) Conceptual block diagram model of the retinal-defocus pathway for regulating sclera growth. The difference between the center and surround excitation provides the retinal-defocus signal. The derivative of the signal drives the release of neuromodulators, which provides the feedback via interplexiform neurons to regulated horizontal cell gain. In addition, release of neuromodulators cause changes in the rate of proteoglycan synthesis, and in turn relative scleral growth rate. (b) Detail block diagram model depicting the regulation of scleral growth rate. The retinal layers (outer to inner) are arranged from left to right: photoreceptor, outer plexiform, bipolar, inner plexiform, and ganglion. The sustained pathway consists of center photoreceptor, center bipolar B and sustained ganglion cell. Horizontal cells, whose gains are regulated by feedback via interplexiform cells, relay surround information to modify sustained ganglion output. The transient pathway consists of center photoreceptor, center bipolar A, and transient ganglion cell. Amacrine cells relay change in surround information to modify transient ganglion output. Center bipolar B signal consists of retinal-defocus information and passes through a rectifier, low-pass filters, and elements representing neuromodulator release, the choroid, and proteoglycan synthesis. This is inverted to provide relative scleral growth rate relative to normal. 
ness (Hung and Ciuffreda, 2000b; Troilo et al., 2000a). This may explain why, as expected, the choroidal thickness changes in the monkey are correlated with changes in retinal-defocus magnitude, but the optical change associated the thickness change is too small to account for any significant contribution towards full emmetropization (Hung and Ciuffreda, 2000b; Troilo et al., 2000a). On the other hand, the neuromodulator that reaches the sclera modifies proteoglycan synthesis to result in changes in ocular growth that does provide nearly full emmetropization, as described in the schematic model above.

Model simulation responses to center and surround stimuli are shown in Fig. 7(a)-(d). The center stimulus, representing sharp focus, consists of a \pm 1 amplitude ptp, $0.1 \mathrm{~Hz}$, square-wave signal. The surround stimuli, representing varying degrees of retinal-image defocus, consists of the same square wave but modulated by different step levels over the time span of the simulation. Figure 7(a) shows the various steps of modulation of the surround amplitude (solid) and the feedback-regulated change in the output of the horizontal cells. As noted above, this provides relatively constant sensitivity to changes in retinal-defocus magnitude. The pulse-like responses for the rates of neuromodulator release (solid) and proteoglycan synthesis (dashed) are shown in Fig. 7(b). The change in proteoglycan synthesis rate in turn causes changes in the scleral growth rate relative to normal [Fig. 7(c)]. Finally, the cumulative change in axial length relative to normal is shown in Fig. 7(d). These results indicate that the model is able to simulate the bi-directional aspects of scleral axial length changes found experimentally, which is consistent with the schematic model provided above.

\section{Summary AND CONCLUSIONS}

Our schematic analysis provides a relatively simple and logically consistent explanation for the eye's ability to grow in the appropriate direction following a lensinduced change in retinal-image contrast. It has also been able to explain the effects of diffusers, black occluder, removal of the crystalline lens, and prolonged nearwork on ocular growth. The critical point is that the detection mechanism does not depend on the sign of the blur, but rather on the change in blur magnitude during genetically-programmed ocular growth. And, it is not necessary to invoke more complicated processes, such as sensing and analysing of chromatic aberration, spherical aberration, spatial gradient of blur, or spatial frequency content (Ciuffreda, 1991, 1998). Thus, our unifying theory provides increased understanding of the underlying retinal mechanisms for detecting blur magnitude. Furthermore, it explains how this signal is processed to modulate the rate of eye growth, and in turn the resultant development of axial myopia. Finally, a MATLAB/SIMULNK model of the retinal pathway for regulating ocular growth demonstrates quantitatively how change in retinal-defocus magnitude can result in appropriate changes in axial growth. 

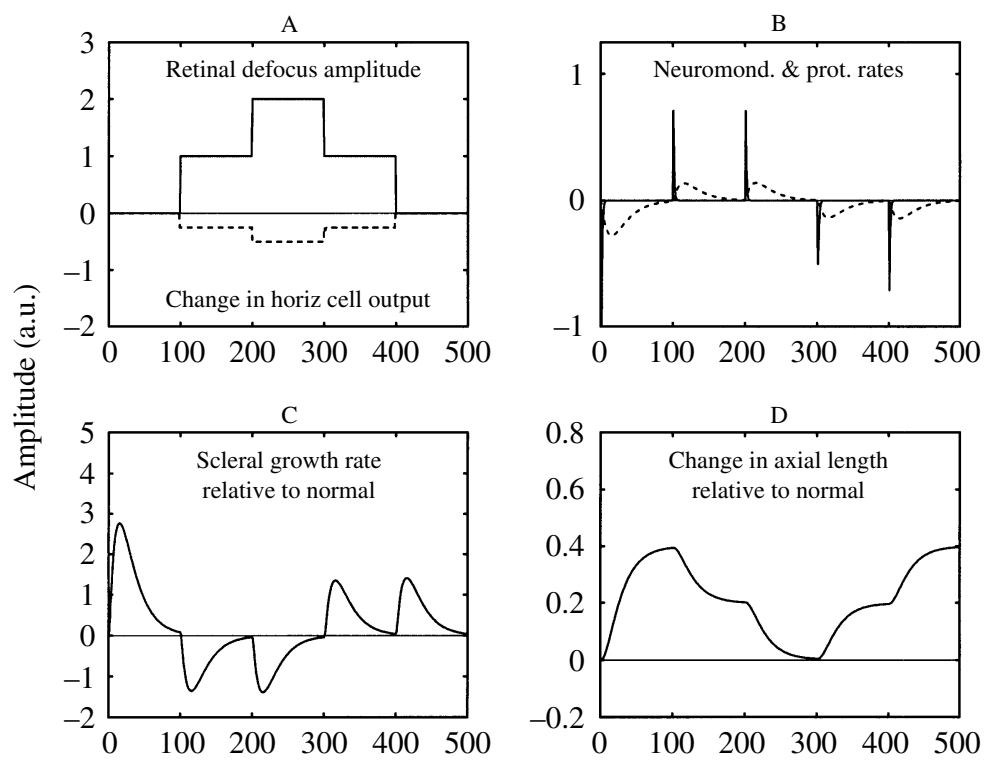

Time (hr)

Figure 7. (a) Envelope of surround stimulus representing various levels of defocus (solid). Changes in horizontal cell output, which is regulated by interplexiform neuronal feedback, shows a complementary response to surround defocus (dashed). (b) Pulses of rates of neuromodulator release (solid) and proteoglycan synthesis (dashed) occur at the transitions of surround stimulus [see (a)]. The initial negative pulse (dashed) is due to model parameter start-up transient response to a small retinal-defocus magnitude. (c) Rate of scleral growth relative to normal follows the pulses in the rate of proteoglycan synthesis. (d) Integration of scleral growth rate provides the change in axial length relative to normal. The direction of change is consistent with experimental results and with the analysis provided by the schematic model. 


\section{ACKNOWLEDGEMENT}

Supported in part by NIH T35 Grant-EY07079.

\section{REFERENCES}

Abbott, M. L., K. L. Schmid and N. C. Strang (1998). Differences in the accommodation stimulus response curves of adult myopes and emmetropes. Ophthal. Physiol. Opt. 18, $13-20$.

Adams, D. W. and N. A. McBrien (1992). Prevalence of myopia and myopic progression in a population of clinical microscopists. Optom. Vis. Sci. 69, 467-473.

Bartmann, M. and F. Schaeffel (1994). A simple mechanism for emmetropization without cues from accommodation or colour. Vision Res. 34, 873-876.

Bennett, A. G. and R. B. Rabbetts (1989). Clinical Visual Optics, Woburn, MA: Butterworth-Heinemann, p. 75.

Bjelke, B. et al. (1996). Dopaminergic transmission in the rat retina: evidence for volume transmission. Chem. Neuroanat. 12, 37-50.

Blackie, C. A. and H. C. Howland (1999). Extension of the Flitcroft model of emmetropization: inclusion of pupil size. Ophthalmic Physiol. Opt. 19, 112-125.

Bradley, D. V., A. Fernandes, M. Tigges and R. G. Boothe (1996). Diffuser contact lenses retard axial elongation in infant rhesus monkeys. Vision Res. 36, 509-514.

Christiansen, A. M. and J. Wallman (1991). Evidence that increased scleral growth underlies visual deprivation myopia in chicks. Invest. Ophthalmol. Vis. Sci. 32, 2134-2150.

Ciuffreda, K. J. (1991). Accommodation and its anomalies, in Vision and Visual Dysfunction: Visual Optics and Instrumentation, Vol. 1, W. N. Charman (Ed.), London: Macmillan, pp. 231-279.

Ciuffreda, K. J. (1998). Accommodation, pupil, and presbyopia, in Borish's Clinical Refraction, W. J. Benjamin (Ed.), Philadelphia, PA: W. B. Saunders Co, pp. 77-120.

Ciuffreda, K. J. and R. V. Kenyon (1983). Accommodative vergence and accommodation in normals, amblyopes, and strabismics, in Vergence Eye Movements: Basic and Clinical Aspects, C. M. Schor and K. J. Ciuffreda (Eds), Boston, MA: Butterworths, pp. 101173.

Cohen, A. I. (1981). The retina and the optic nerve, in Adler's Physiology of the Eye, Clinical Applications, R. A. Moses (Ed.), St. Louis, MO: C. V. Mosby Company, pp. 370410.

Dowling, J. E. (1996). Retinal processing of vision, in Comprehensive Human Physiology: From Cellular Mechanisms to Integration, Vol. 1, R. Greger and U. Windhorst (Eds), Berlin: Springer-Verlag, pp. 773-778.

Fledelius, H. C. and M. Stubgaard (1986). Changes in refraction and corneal curvature during growth and adult life. A cross-sectional study. Acta Ophthalmol. 64, 487-491.

Flitcroft, D. I. (1998). A model of the contribution of oculomotor and optical factors to emmetropization and myopia. Vision Res. 38, 2869-2879. 
Garner, L. F., C. K. Meng, T. P. Grosvenor and N. Mohidin (1990). Ocular dimensions and refractive power in Malay and Melanesian children. Ophthalmic Physiol. Opt. 10, 234-238.

Goss, D. A. and P. Erickson (1987). Meridional corneal components of myopia progression in young adults and children. Am. J. Optom. Physiol. Opt. 64, 475-481.

Goss, D. A. and T. W. Jackson (1993). Cross-sectional study of changes in the ocular components in school children. Appl. Opt. 32, 4169-4173.

Goss, D. A. and M. G. Wickham (1995). Retinal-image mediated ocular growth as a mechanism for juvenile onset myopia and for emmetropization. Doc. Ophthalmol. 90, 341375.

Goss, D. A. and R. L. Winkler (1983). Progression of myopia in youth: age of cessation. Am. J. Optom. Physiol. Opt. 60, 651-658.

Gottlieb, M. D., H. B. Joshi and D. L. Nickla (1990). Scleral changes in chicks with formdeprived myopia. Curr. Eye Res. 9, 1157-1165.

Grosvenor, T. and D. A. Goss (1998). Role of the cornea in emmetropia and myopia. Optom. Vis. Sci. 75, 132-145.

Grosvenor, T. and D. A. Goss (1999). Etiology of myopia, in Clinical Management of Myopia, Boston, MA: Butterworth-Heinemann, pp. 49-62.

Gwiazda, J., F. Thorn, J. Bauer and R. Held (1993). Emmetropization and the progression of manifest refraction in children followed from infancy to puberty. Clin. Vis. Sci. 8 , 337-344.

Hung, G. K. (1998). Sensitivity analysis of the stimulus-response function of a static nonlinear accommodation model. IEEE Trans. Biomed. Eng. 45, 335-341.

Hung, G. K. and K. J. Ciuffreda (1999). Model of refractive error development. Curr. Eye Res. 19, 41-52.

Hung, G. K. and K. J. Ciuffreda (2000a). Differential retinal-defocus magnitude during eye growth provides the appropriate direction signal. Med. Sci. Monitor 6, 791-795.

Hung, G. K. and K. J. Ciuffreda (2000b). Quantitative analysis of the effect of near lens addition on accommodation and myopigenesis. Curr. Eye Res. 20, 293-312.

Hung, L. F., J. Wallman and E. L. Smith (2000b). Vision-dependent changes in the choroidal thickness of Macaque monkeys. Invest. Ophthalmol Vis. Sci. 41, 1259-1269.

Iuvone, P. M., M. Tigges, R. A. Stone, S. Lambert and A. M. Laties (1991). Effect of apomorphine, a dopamine receptor agonist, on ocular refraction and axial elongation in primate model of myopia. Invest. Ophthalmol Vis. Sci. 32, 1674-1677.

Jiang, B. C. and W. M. Woessner (1996). Increase in axial length is responsible for lateonset myopia. Optom. Vis. Sci. 73, 231-234.

Lin, L. L. K., Y. F. Shih, Y. C. Lee, P. T. Hung and P. K. Hou (1996). Changes in ocular refraction and its components among medical students-a 5-year longitudinal study. Optom. Vis. Sci. 73, 495-498.

Marzani, D. and J. Wallman (1997). Growth of the two layers of the chick sclera is modulated reciprocally by visual conditions. Invest. Ophthalmol. Vis. Sci. 38, 1726-1739. 
McBrien, N. A., A. Gentle and C. Cottriall (1999). Optical correction of induced axial myopia in the tree shrew: implications for emmetropization. Optom. Vis. Sci. 76, 419427.

McBrien, N. A. and M. Millodot (1986). The effect of refractive error on the accommodative response gradient. Ophthalmic Physiol. Opt. 6, 145-149.

Medina, A. (1987). A model of emmetropization, the effect of corrective lenses. Acta Ophthalmol. 65, 585-571.

Norton, T. T. (1999). Animal models of myopia: learning how vision controls the size of the eye. Instit. Lab. Animal Res. J. 40, 59-77.

Norton, T. T. and J. A. Rada (1995). Reduced extracellular matrix in mammalian sclera with induced myopia. Vision Res. 35, 1271-1281.

O'Leary, D. J., K. M. Chung and S. Othman (1992). Contrast reduction without myopia induction in monkey. Invest. Ophthalmol. Vis. Sci. 33 (Suppl.), 712.

Ong, E. and K. J. Ciuffreda (1997). Accommodation, Nearwork, and Myopia: Optometric Extension Program Foundation, Inc, Santa Ana, CA, pp. 76-96, 177-201.

Ong, E., K. J. Ciuffreda and B. Tannen (1993). Static accommodation in congenital nystagmus. Invest. Ophthalmol. Vis. Sci. 34, 194-204.

Rada, J. A., A. L. McFarland, P. K. Cornuet and J. R. Hassell (1992). Proteoglycan synthesis by scleral chondrocytes is modulated by a vision dependent mechanism. Curr. Eye Res. 11, 767-782.

Rosenfield, M. and B. Gilmartin (1998). Myopia and nearwork: causation or merely association? in Myopia and Nearwork, M. Rosenfield and B. Gilmartin (Eds), Oxford: Butterworth-Heinemann, pp. 193-206.

Scammon, R. E. and E. L. Armstrong (1925). On the growth of the human eyeball and optic nerve. J. Comp. Neurol. 38, 165-219.

Schaeffel, F. and H. C. Howland (1988). Mathematical model of emmetropization in the chicken. J. Opt. Soc. Am. A 5, 2080-2086.

Schaeffel, F., D. Troilo, J. Wallman and H. C. Howland (1990). Developing eyes that lack accommodation grow to compensate for imposed defocus. Vis. Neurosci. 4, 177-183.

Siegwart, J. T. Jr. and T. T. Norton (1998). A susceptible period for deprivation-induced myopia in tree shrew. Vision Res. 38, 3505-3515.

Siegwart, J. T. Jr. and T. T. Norton (1999). Regulation of the mechanical properties of tree shrew sclera by the visual environment. Vision Res. 39, 387-407.

Smith, E. L. and L. F. Hung (1999). The role of optical defocus in regulating refractive development in infant monkeys. Vision Res. 39, 1415-1435.

Smith, E. L. and L. F. Hung (2000). Form deprivation myopia in monkeys is a graded phenomenon. Vision Res. 40, 371-381.

Smith, G. and D. A. Atchison (1997). The Eye and Visual Optical Instruments, Cambridge, United Kingdom: Cambridge University Press, pp. 274, 796.

Sorsby, A., B. Benjamin and M. Sheridan (1961). Refraction and its Components During the Growth of the Eye from the Age of Three, Med Res Council Report Series 301, London: Her Majesty's Stationery Office.

Sperduto, R. D., D. Seigel, J. Roberts and M. Rowland (1983). Prevalence of myopia in the United States. Arch. Ophthalmol. 101, 405-407. 
Stark, L. (1968). Neurological Control Systems, Studies in Bioengineering, New York: Plenum Press, pp. 205-219.

Stone, R. A., T. Lin and A. M. Laties (1989). Retinal dopamine and form-deprivation myopia. Proc. Natl. Acad. Sci. 86, 704-706.

Tigges, M., J. Tigges, A. Fernendes, H. M. Effers and J. A. Gammon (1990). Postnatal axial eye elongation in normal and visually deprived rhesus monkeys. Invest. Ophthalmol. Vis. Sci. 31, 1035-1046.

Troilo, D. (1989). The visual control of eye growth in chicks, PhD dissertation, City College of the City University of New York, New York.

Troilo, D., M. D. Gottlieb and J. Wallman (1987). Visual deprivation causes myopia in chicks with optic nerve section. Curr. Eye Res. 6, 993-999.

Troilo, D., D. L. Nickla and J. Wallman (2000a). Choroidal thickness changes during altered eye growth and refractive state in a primate. Invest. Ophthalmol. Vis. Sci. 41, 12491258.

Troilo, D., D. L. Nickla and C. F. Wildsoet (2000b). Form deprivation myopia in mature common Marmoset (Callitbrix jaccbus). Invest. Ophthalmol. Vis. Sci. 41, 2043-2049.

Van Alphen, G. W. (1961). On emmetropia and ametropia. Acta Ophthalmol 142 (Suppl.), Karger, Basel, pp. 1-92.

Wallman, J. (1997). Can myopia be prevented? in $14^{\text {th }}$ Biennial Research to Prevent Blindness Science Writers Seminar in Ophthalmology, New York: Research to Prevent Blindness, pp. 50-52.

Weale, R. A. (1982). A Biography of the Eye: Development, Growth, Age, London: H. K. Lewis.

Westheimer, G. (1981). Visual acuity, in Adler's Physiology of the Eye, R. A. Moses (Ed.), St. Louis: C.V. Mosby Co., pp. 530-544.

Wildsoet, C. F. (1998). Structural correlates of myopia, in Myopia and Nearwork, M. Rosenfield and B. Gilmartin (Eds), Oxford: Butterworth-Heinemann, pp. 32-51.

Wildsoet, C. F. and M. J. Collins (2000). Competing defocus stimuli of opposite sign produce opposite effects in eyes with intact and section optic nerves in the chick. Invest. Ophthalmol. Vis. Sci. 41, S738.

Wildsoet, C. F. and J. D. Pettigrew (1988). Experimental myopia and anomalous eye growth patterns unaffected by optic nerve section in chickens: Evidence for local control of eye growth. Clin. Vis. Sci. 3, 99-107.

Wilson, J. R., A. Fernandes, C. V. Chankler, M. Tigges, R. G. Boothe and J. A. Gammon (1987). Abnormal development of the axial length of aphakic monkey eyes. Invest. Ophthalmol. Vis. Sci. 28, 2096-2099.

Winauer, J. A., X. Zhu, T. Park and J. Wallman (2000). Is myopic blur more important than sharp vision for positive-lens compensation? Invest. Ophthalmol. Vis. Sci. 41, S136.

Windhorst, U. (1996). Specific networks of the cerebral cortex: functional organization and plasticity, in Comprehensive Human Physiology: From Cellular Mechanisms to Integration, Vol. 1, R. Greger and U. Windhorst (Eds), Berlin: Springer-Verlag, pp. 1105-1136. Yackle, K. and D. E. Fitzgerald (1999). Emmetropization: an overview. J. Behav. Optom. 10, 38-43. 
York, M. A. and R. B. Mandell (1969). A new calibration system for photokeratoscopy. II. Corneal contour measurements. Am. J. Optom. Arch. Am. Acad. Optom. 46, 818-825.

Received 2 February 2000 and accepted 25 June 2000 